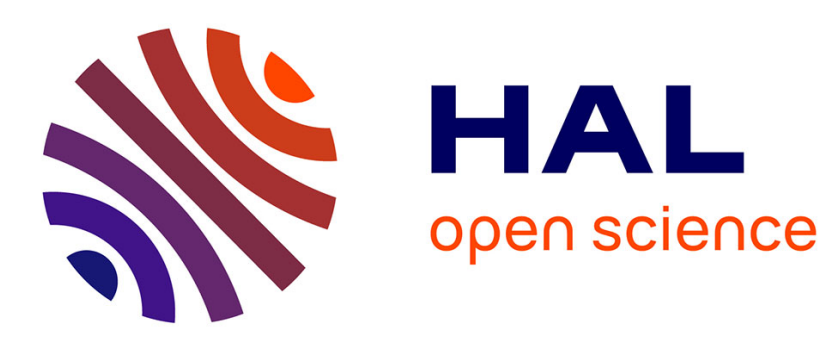

\title{
Comptes intégrés économiques et environnementaux de la forêt en France. Première partie
}

\author{
Claire Montagné, Alexandra Niedzwiedz
}

\section{To cite this version:}

Claire Montagné, Alexandra Niedzwiedz. Comptes intégrés économiques et environnementaux de la forêt en France. Première partie. Revue forestière française, 2010, 62 (5), pp.541-550. $10.4267 / 2042 / 39865$. hal-03449623

\section{HAL Id: hal-03449623 https://hal.science/hal-03449623}

Submitted on 25 Nov 2021

HAL is a multi-disciplinary open access archive for the deposit and dissemination of scientific research documents, whether they are published or not. The documents may come from teaching and research institutions in France or abroad, or from public or private research centers.
L'archive ouverte pluridisciplinaire HAL, est destinée au dépôt et à la diffusion de documents scientifiques de niveau recherche, publiés ou non, émanant des établissements d'enseignement et de recherche français ou étrangers, des laboratoires publics ou privés. 


\title{
hCtIUITES, PRODUITS Et mahChES
}

\section{COMPTES INTÉGRÉS \\ ÉCONOMIQUES ET ENVIRONNEMENTAUX \\ DE LA FORÊT EN FRANCE}

Première partie

\section{Claire Montagné - Alexandra Niedzwiedz}

\begin{abstract}
Avertissement : Cet article présente les concepts généraux et l'expérience française en matière de comptabilité forestière. En France, les comptes de la forêt sont réalisés par le Laboratoire d'économie forestière de Nancy (INRA - AgroParisTech ENGREF) en partenariat avec l'Inventaire forestier national et les principaux organismes producteurs de données sur la forêt et le bois. Ces comptes, réalisés selon le format européen défini par Eurostat (direction générale de la Commission européenne chargée de l'information statistique à l'échelle communautaire), ont pour objectif de refléter les valeurs liées à la forêt à partir d'indicateurs physiques et monétaires en intégrant au maximum les multiples biens et services fournis par les forêts. Ils sont constitués de 13 tableaux comptables (European Commission, 2002a, 2002b) :

- Stocks et flux physiques des forêts françaises en surface (1 ooo ha)

- Valeur du fonds des surfaces boisées en France (en million d'euros)

- Volume de bois sur pied (1 00o m³)

- Valeur du bois sur pied (en million d'euros)

- Défoliation (\% arbres)

- Production des surfaces boisées par industries et type de production (en million d'euros)

- Comptes de la sylviculture et de l'exploitation forestière (en million d'euros)

- Emplois du bois dans le secteur forestier en unités physiques

- Ressources du bois dans le secteur forestier en unités physiques

- Emplois du bois dans le secteur forestier en unités monétaires

- Ressources du bois dans le secteur forestier en unités monétaires

- Bilan carbone de la biomasse forestière (1 ooo tonnes de carbone)

- Bilan carbone de l'écosystème forestier (1 ooo tonnes de carbone)
\end{abstract}

Cet article est publié en deux parties dans deux numéros successifs de la Revue forestière française. La première partie présente le cadre général des comptes de la forêt, l'analyse comptable du patrimoine forestier (surfaces, volumes, état sanitaire) et les comptes en carbone (biomasse et écosystème forestiers) ; la seconde partie, qui sera publiée dans le numéro 6-2010, présentera l'analyse comptable du secteur forestier et des activités qui lui sont liées.

\section{LA COMPTABILITÉ NATIONALE}

La comptabilité nationale consiste à mesurer l'ensemble des flux monétaires caractérisant l'activité économique d'un pays. Il en émane une représentation cohérente de l'économie par activité 
et des indicateurs économiques incontournables comme le produit intérieur brut (PIB). Cependant, le système comptable traditionnel peine à décrire les liens entre économie et environnement : alors que la forêt française compte parmi les plus importantes et les plus diverses de l'Union européenne, le secteur forestier français occupe une position très marginale dans l'économie nationale : 0,8 \% du PIB en 2005 (CMPFE, 2007). Une comptabilité forestière spécifique permet de porter un regard plus objectif sur la multifonctionnalité des espaces boisés et d'assurer un meilleur suivi des ressources et de l'économie du bois.

En outre, comme le préconisent Stiglitz et al. (2009), la mesure des performances économiques et du progrès social nécessite la prise en compte d'indicateurs physiques des pressions sur l'environnement. Une solution est la réalisation de comptes satellites de l'environnement (United Nations, 1993, 2003) tels que les comptes intégrés économiques et environnementaux de la forêt (Integrated Environmental and Economic Accountings for Forest, IEEAF) qui sont une illustration de la façon dont ces préoccupations peuvent être introduites dans un système de comptabilité sectorielle. Ils permettent de prendre en compte d'autres éléments, comme le capital naturel, sans altérer la cohérence de la comptabilité nationale. En effet, si la comptabilité nationale préconise des évaluations monétaires fondées sur le marché pour l'ensemble des biens économiques, il se peut que pour certains biens ou services produits par la forêt il n'en existe pas. La comptabilité forestière tente d'évaluer l'ensemble des biens et services forestiers, qu'ils soient marchands ou non. C'est le cas pour les services de protection rendus par la forêt ou la récréation : il n'existe pas de marché sur lequel fonder des évaluations économiques. La nécessité est donc apparue de trouver d'autres méthodes d'évaluation, comme l'évaluation contingente, pouvant être utilisées pour la comptabilité nationale. Ces méthodes attribuent une valeur à un bien ou un service, faisant l'hypothèse qu'ils peuvent avoir une valeur économique si les acteurs économiques étaient prêts à les payer. Ce type de comptabilité tente d'estimer le véritable potentiel du patrimoine forestier, qui ne se limite pas à la production de bois.

Les IEEAF, initiés dès 1995 par l'Office statistique des Communautés européennes (Eurostat), s'inscrivent dans ce cadre et représentent la structure méthodologique de la comptabilité forestière au niveau européen (European Commission, 2002a). Depuis 1997, le Laboratoire d'économie forestière (LEF) et les principaux organismes professionnels et statistiques français - le Service de l'observation et des statistiques du ministère de l'Écologie, du Développement durable, des Transports et du Logement (SOeS), le Service de la statistique et de la prospective du ministère de l'Agriculture, de l'Alimentation, de la Pêche, de la Ruralité et de l'Aménagement du Territoire (SSP), l'Institut national de la statistique et des études économiques (INSEE), l'Inventaire forestier national (IFN) - participent à cette réflexion. Des années de travail ont débouché sur un ensemble de comptes quinquennaux et annuels sur la période 1981-2006 (European Commission, 2002b ; Montagné et Niedzwiedz, 2009).

Les IEEAF sont comme une passerelle liant le monde forestier à la réalité économique. Ils ont pour objectif de mieux mesurer les interactions entre économie et environnement en dressant à la fois des bilans physiques (environnementaux) et monétaires (économiques) du secteur forestier et en les mettant en cohérence. Les comptes de la forêt décrivent le patrimoine naturel forestier, ses multiples usages et les flux de biens et services qui en découlent.

Ainsi, les comptes de patrimoine décrivent l'état de la ressource forestière (surfaces, volumes, état sanitaire) ; les comptes du secteur forestier (1) comptabilisent l'ensemble des inputs et outputs liés à la forêt (compte de la production forestière, compte de la sylviculture et de

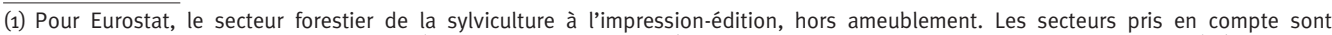
donc : sylviculture, exploitation forestière (code 02 de la NACE rev.1), le travail du bois et fabrication d'articles en bois (20), industrie de la pâte (21.11), industrie du papier (21.12 et 21.2), impression (22), recyclage et gestion des déchets (partie des codes 37.2 et 90 ). 


\begin{tabular}{|c|c|c|c|c|}
\hline \multirow{2}{*}{\multicolumn{2}{|c|}{ Activité/Produits }} & \multicolumn{2}{|c|}{ Données intérieures } & \multirow{2}{*}{$\begin{array}{c}\begin{array}{c}\text { Commerce } \\
\text { extérieur }\end{array} \\
\begin{array}{c}\text { Physiques } \\
\text { et monétaires }\end{array}\end{array}$} \\
\hline & & & & \\
\hline \multirow[t]{3}{*}{ Forêt. . . . . . . } & Surfaces & IFN/LEF ${ }^{a}$ & LEF/FNSAFER ${ }^{k}$ & \\
\hline & Volumes & IFN/LEF & ONF/LEF ${ }^{l}$ & \\
\hline & $\begin{array}{l}\text { Fonctions non-bois } \\
\text { Carbone } \\
\text { Récréation } \\
\text { Protection } \\
\text { Défoliation } \\
\text { Venaison } \\
\text { Liège } \\
\text { Autres services }\end{array}$ & $\begin{array}{c}\text { CARBOFOR }{ }^{\mathrm{b}} \text { Lochu }^{\mathrm{c}} \\
\mathrm{LEF}^{\mathrm{d}} \\
\mathrm{IFN}^{\mathrm{e}} \\
\mathrm{DSF}^{\mathrm{f}}\end{array}$ & $\begin{array}{c}\text { LEF } \\
\text { LEF } \\
\text { MAAPRAT }^{\mathrm{m}} \text {, ONF }^{\mathrm{n}}, \text { MIOCTI }^{\circ} \\
\text { ONCFS }^{p} \\
\text { CRPF PACA }^{\mathrm{q}}, \text { IML }^{\mathrm{r}} \text {, ODARC } \\
\text { ONF, IFN, DSF, MAAPRAT, } \\
\text { MIOCTI }\end{array}$ & \\
\hline Récolte.... . & Bois ronds & Agreste EAB-EFS ${ }^{g}$ & $\begin{array}{l}\text { ONF/LEF } \\
\text { MAAPRAT }\end{array}$ & \multirow[t]{7}{*}{ Douanes/SSPv } \\
\hline \multirow[t]{3}{*}{$\begin{array}{l}\text { Première } \\
\text { transformation }\end{array}$} & $\begin{array}{l}\text { Placages } \\
\text { et contreplaqués }\end{array}$ & $\mathrm{FAO}^{\mathrm{h}}$ & \multirow[t]{2}{*}{ INSEE $^{\mathrm{t}}$} & \\
\hline & Sciages & Agreste EAB-EFS & & \\
\hline & $\begin{array}{l}\text { Produits connexes } \\
\text { de scierie }\end{array}$ & Agreste EAB-EFS & $\mathrm{FCBA}^{\mathrm{u}}$ & \\
\hline \multirow{3}{*}{$\begin{array}{l}\text { Seconde } \\
\text { transformation }\end{array}$} & Panneaux & FAO & \multirow{3}{*}{ INSEE } & \\
\hline & $\begin{array}{l}\text { Pâte à papier, papiers, } \\
\text { cartons, vieux papiers }\end{array}$ & COPACEL ${ }^{\mathrm{i}}$ & & \\
\hline & Autres produits & $E A B^{j}$ & & \\
\hline
\end{tabular}

a IFN/LEF : Données de l'Inventaire forestier national retravaillées par le Laboratoire d'économie forestière

${ }^{b}$ CARBOFOR : Coefficients issus du projet multidisciplinaire CARBOFOR (GICC, 2004)

' Lochu : Coefficients issus du rapport Lochu (1998)

d LEF : Données issues d'enquêtes ou travaux réalisés par le Laboratoire d'économie forestière

e IFN : Inventaire forestier national

${ }^{\mathrm{f}}$ DSF : Département de la santé des forêts du ministère de l'Agriculture, de l'Alimentation, de la Pêche, de la Ruralité et de l'Aménagement du Territoire

${ }^{\mathrm{g}}$ Agreste EAB-EFS : Enquête annuelle de branche exploitation forestière et scieries du SSP

${ }^{\text {h }}$ FAO : Organisation des Nations unies pour l'alimentation et l'agriculture

i COPACEL : Confédération française de l'industrie des papiers cartons et celluloses

¡ EAB : Enquête annuelle de branche (Service des études et des statistiques industrielles de l'INSEE)

k LEF/FNSAFER : Indice de la Fédération nationale des sociétés d'aménagement foncier de l'espace rural appliqué

à une donnée de référence $L E F$

' ONF/LEF : Matrice de passage LEF appliquée aux prix de l'Office national des forêts

${ }^{m}$ MAAPRAT : Ministère de l'Agriculture, de l'Alimentation, de la Pêche, de la Ruralité et de l'Aménagement du Territoire

${ }^{n}$ ONF : Office national des forêts

${ }^{\circ}$ MIOCTI : Ministère de l'Intérieur, de l'Outre-mer, des Collectivités territoriales et de l'Immigration

P ONCFS : Office national de la chasse et de la faune sauvage

q CRPF PACA : Centre régional de la propriété forestière Provence-Alpes-Côte d’Azur

$r$ IML : Institut méditerranéen du liège

s ODARC : Office du développement agricole et rural de Corse

${ }^{t}$ INSEE : Institut national de la statistique et des études économiques

u FCBA : Institut technologique forêt cellulose bois-construction ameublement

${ }^{\vee}$ Douanes/SSP : Données des douanes reprises dans le commerce extérieur des bois et dérivés du SSP 
l'exploitation forestière, tableaux emplois-ressources) et dressent une analyse fonctionnelle des transactions ; les comptes en carbone mesurent le carbone stocké dans la biomasse et l'écosystème forestiers.

En introduisant les concepts comptables à la statistique environnementale, les IEEAF constituent un système d'information articulé, rigoureux, régulier et normalisé, fournissant des indicateurs permettant notamment l'évaluation des politiques publiques. En outre, le cadre imposé par Eurostat est commun à tous les pays membres et permet de ce fait les comparaisons internationales.

Le présent article a choisi de valoriser deux points importants des IEEAF :

- d'une part, le fait que ces comptes sont établis annuellement ;

- d'autre part, le fait qu'ils permettent, malgré un cadre strict, la prise en compte d'événements exceptionnels majeurs tels que les tempêtes.

Malgré tout, si les 13 tableaux sont formalisés par Eurostat, ils peuvent être adaptés en cas de nécessité (pour répondre à des spécificités nationales par exemple) ou détaillés selon les possibilités.

Leur réalisation repose sur un important travail de synthèse et de mise en cohérence des données issues de sources diverses (tableau I, p. 543) et sur l'élaboration de méthodes originales. Le LEF contribue donc à structurer l'information et à pallier le manque de données par des estimations ou des enquêtes.

Dans la suite de l'article, les sources des données seront indiquées par des lettres en exposant, selon la légende du tableau I. Les principales unités et abréviations utilisées sont présentées dans le tableau II.

TABLEAU II Unités, quantités et symboles

\begin{tabular}{|c|c|}
\hline hectare $\ldots \ldots \ldots \ldots \ldots \ldots \ldots \ldots \ldots \ldots$ & ha \\
\hline euro $\ldots \ldots \ldots \ldots \ldots \ldots \ldots \ldots$ & $€$ \\
\hline kilo-, millier $(\times 1000) \ldots \ldots \ldots$. . . . . & $\mathrm{k}$ \\
\hline méga-, million $(\times 1000000) \ldots \ldots \ldots$ & M \\
\hline mètre cube équivalent bois rond . . . . . . & $\mathrm{m}^{3} \mathrm{ebr}$ \\
\hline tonne de carbone .............. & $\mathrm{tC}$ \\
\hline
\end{tabular}

Les unités physiques utilisées diffèrent entre produits ou entre sources. Par exemple, la production de bois rond est exprimée en mètres cubes alors que son commerce extérieur est exprimé en tonnes.

Afin de disposer de données permettant le suivi du bois tout au long de la filière, il est nécessaire de disposer d'une unité physique unique pour tous les produits : le mètre cube équivalent bois rond ( $\mathrm{m}^{3} \mathrm{ebr}$ ). Il s'agit d'exprimer une quantité donnée de produit sous la forme du volume initial de bois rond nécessaire à sa production. Par exemple, il faut, en moyenne, $2 \mathrm{~m}^{3}$ de bois rond pour fabriquer $1 \mathrm{~m}^{3}$ de sciage, ou $4 \mathrm{~m}^{3}$ de bois rond pour fabriquer 1 tonne de contreplaqué. Cette unité permet d'homogénéiser les quantités et rend possible les comparaisons entre produits et la réalisation de bilans matière bois (SSP). 


\section{LES COMPTES DE PATRIMOINE}

Leur objectif est de rendre compte annuellement de l'état de la ressource forestière. Ils reflètent en unités physiques et monétaires les stocks et flux de surface forestière et de volume de bois sur pied, ainsi que l'état sanitaire des forêts.

\section{Les comptes en surface}

Les surfaces forestières sont entendues selon la définition de la FAO (UNECE/FAO TBFRA, 2000). La forêt est un territoire occupant une superficie d'au moins 0,5 ha ayant un couvert de plus de $10 \%$ (forêts). Les autres terres boisées correspondent aux terres de plus de 0,5 ha ayant un couvert arboré de 5 à $10 \%$ d'arbres capables d'atteindre une hauteur de $5 \mathrm{~m}$ à maturité in situ, soit un couvert arboré de plus de $10 \%$ d'arbres qui ne sont pas en mesure d'atteindre une hauteur de $5 \mathrm{~m}$ à maturité in situ. Les formations boisées inférieures à 0,5 ha (dont font partie les bosquets) sont exclues de la définition FAO. Travaillant à partir des données IFN « ancienne méthode ", les bosquets ont cependant été inclus dans les autres terres boisées.

Les " forêts et autres terres boisées » sont distinguées selon qu'elles sont disponibles ou non pour la production de bois. Une forêt de production est une forêt disponible pour la production de bois : l'exploitation du bois y est possible (sans considération de rentabilité économique) et compatible avec d'éventuelles autres fonctions ${ }^{e}$. Ces surfaces sont comptabilisées en unités physiques et monétaires (tableau III, p. 546).

La surface boisée totale à la date de clôture du compte est obtenue à partir de la surface boisée de la période précédente corrigée des variations, naturelles ou d'origine anthropique, enregistrées sur la période.

Les données physiques en surface sont issues de l'IFN. Jusque 2004, l'IFN n'inventoriait qu'une dizaine de départements chaque année. Les données disponibles à une date précise ne reflétaient donc pas l'état réel de la forêt. Le LEF actualisait les données issues de ces inventaires afin de disposer d'une estimation annuelle de l'état de la forêt française. Depuis 2005, l'IFN a mis en place de nouvelles définitions et une nouvelle méthode d'inventaire, qui fournit, à compter de 2010, des données annuelles. Les données présentées ici concernent la période 2000-2006, elles sont obtenues à partir d'estimations réalisées par le LEF. (Les données moyennes des trois premières campagnes du nouvel inventaire de l'IFN sont considérées comme représentatives des surfaces au 31 décembre 2006. Elles sont ensuite "rétropolées » jusqu'en 2000 selon l'évolution annuelle observée sur les 10 dernières années a. Ainsi, nous disposons d'estimations au début et à la fin de chaque période. Ces estimations seront affinées après publication des données annuelles de l'IFN.)

La valeur du fonds des surfaces boisées, c'est-à-dire la valeur du sol nu, est estimée à partir des transactions de marché. La valorisation des terres boisées se fait en appliquant le prix à l'hectare aux surfaces. Ce prix à l'hectare est obtenu à partir d'une valeur de référence, que nous faisons évoluer grâce à l'indice du marché des forêts. Il s'agit de la valeur du fonds forestier pour 1996, issue d'une enquête réalisée par le LEF auprès de la direction générale des impôts (DGI), corrigée d'une évolution annuelle d'après l'indice FNSAFER du marché des forêts.

La ligne réévaluation enregistre les variations dues à des changements de prix à l'hectare entre le début et la fin de période. Il s'agit de la différence entre la valeur des sols forestiers calculée en fin de période avec les nouveaux prix et la valeur que l'on aurait obtenue si les prix avaient été constants. 
TABLEAU III Stocks et flux, physiques et monétaires, des surfaces boisées (2000 et 2006) ${ }^{\mathrm{a}, \mathrm{k}}$

\begin{tabular}{|c|c|c|c|c|c|c|}
\hline & \multicolumn{6}{|c|}{ Forêts et autres terres boisées } \\
\hline & \multicolumn{3}{|c|}{$\begin{array}{l}\text { Surface boisée } \\
\times 1000 \text { ha }\end{array}$} & \multicolumn{3}{|c|}{$\begin{array}{l}\text { Valeur du fonds } \\
\text { M€ courants }\end{array}$} \\
\hline & $\begin{array}{l}\text { Forêts de } \\
\text { production }\end{array}$ & $\begin{array}{c}\text { Autres forêts et } \\
\text { terres boisées }\end{array}$ & Total & $\begin{array}{l}\text { Forêts de } \\
\text { production }\end{array}$ & $\begin{array}{c}\text { Autres forêts et } \\
\text { terres boisées }\end{array}$ & Total \\
\hline Stock au 01/01/2000 $\ldots \ldots$ & 14689 & 956 & 15645 & 14068 & 916 & 14984 \\
\hline $\begin{array}{l}\text { Variations liées aux activités } \\
\text { économiques. . . . . . . . }\end{array}$ & 1 & 0 & 1 & 1 & 0 & 1 \\
\hline Boisement. . . . . . . . . . . & 72 & 5 & 77 & 69 & 4 & 74 \\
\hline Déboisement. . . . . . . . . & -71 & -5 & -76 & -68 & -4 & -73 \\
\hline Variations naturelles. . . . . . & 54 & 3 & 58 & 52 & 3 & 55 \\
\hline Colonisation naturelle. . . . . . & 68 & 4 & 72 & 65 & 4 & 69 \\
\hline Régression naturelle. . . . . . & -11 & 1 & -12 & -11 & -1 & -11 \\
\hline Autre $\ldots \ldots \ldots \ldots$ & -2 & 0 & -2 & -2 & 0 & -2 \\
\hline Réévaluation . . . . . . . . . & - & - & - & 411 & 27 & 438 \\
\hline Stock au $31 / 12 / 2000 \ldots \ldots$ & 14744 & 960 & 15704 & 14532 & 946 & 15477 \\
\hline Stock au 01/01/2006 $\ldots \ldots$ & 15022 & 925 & 15947 & 16389 & 1009 & 17398 \\
\hline Réévaluation . . . . . . . . . . & - & - & - & 2069 & 125 & 2194 \\
\hline Stock au $31 / 12 / 2006 \ldots \ldots$ & 15079 & 909 & 15987 & 18519 & 1116 & 19636 \\
\hline
\end{tabular}

Note 1 : Suite au changement d'enquête TERUTI, le détail de l'origine des flux n'est pas disponible de 2004 à 2006.

Note 2 : Les valeurs étant arrondies, certains totaux sont seulement approchés.

La superficie totale des terres boisées françaises est de 16 millions d'hectares en 2006 : elle couvre $29 \%$ du territoire. Près de $95 \%$ de ces surfaces sont des forêts de production.

Alors que ni les données IFN, ni les séries TERUTI, du fait des changements d'échantillons, de nomenclatures et de méthodes entre 2004 et 2006, ne permettent de disposer de séries homogènes sur 2000-2006, les comptes de la forêt autorisent l'analyse des surfaces sur cette période. D’après nos estimations, la surface des terres boisées a globalement augmenté de 0,31 \%/an (environ 49000 ha/an) entre 2000 et 2006. L'augmentation estimée des surfaces de forêts de production est de $0,37 \% / a n$, soit un gain moyen annuel de 55000 ha, alors que les forêts non productives régressent de 0,7\%/an sur la période 2000-2006. Les bosquets, inclus dans ces surfaces " non productives ", observent une forte diminution $(-4,5 \% / a n)$ et leur surface est équivalente à celle des peupleraies.

Le rythme d'accroissement des surfaces forestières s'est ralenti entre les périodes 1990-2000 et 2000-2006. On constate un ralentissement des boisements depuis 2000, notamment dû à la diminution des aides publiques pour le boisement de terres agricoles. De plus, la loi d'orientation sur la forêt de 2001 (République française, 2001) privilégie le regroupement et l'amélioration des peuplements forestiers existants plutôt que la création de nouveaux espaces boisés.

L'évolution de la valeur des surfaces forestières s'explique par l'augmentation des surfaces et par l'augmentation des prix du fonds forestier (moins de 1000 euros/ha en 2000 et plus de 1200 euros/ha en $2006^{\mathrm{d}, \mathrm{k}}$ ). 
La valeur du fonds forestier s'est accrue de $28 \%$ entre 2000 et 2006 (en moyenne 3,6 \%/an). Cette hausse continue n'a été que faiblement ralentie par les tempêtes de 1999 et par le rétablissement des droits de mutation en 2005. (Les droits de mutation avaient été supprimés en 2000 pour soutenir le marché après les deux tempêtes de $1999^{\mathrm{k}}$.)

La hausse des prix provient en partie de l'inflation immobilière. On peut aussi penser que les efforts consentis pour améliorer la perception de la forêt ont eu un effet positif sur les prix. La forêt est davantage perçue comme un bien potentiellement rémunérateur, comme un placement de long terme et la mise en place de marchés du carbone ouvre de nouvelles perspectives de revenus. La forêt est enfin un outil de production; sa valeur suit la demande de produits bois qui semble s’accroître avec la prise de conscience écologique et l'apparition de marchés émergents.

\section{Les comptes en volume}

Ces comptes en volume décrivent, en unités physiques et monétaires, les variations observées $\mathrm{du}$ volume de bois sur pied entre l'ouverture et la fermeture du compte (tableau IV, p. 548).

En France, seuls les volumes de bois des forêts de production inventoriés par l'IFN sont comptabilisés, en volume bois fort branches sur écorce. [Les comptes sont d'abord réalisés en volume bois fort tige pour les années 2006, 2000 et 2001. En 2006, le stock de fermeture et la production brute sont obtenus à partir des données moyennes des trois premiers inventaires de l'IFN nouvelle méthode. Pour 2000 et 2001, ils sont estimés par le LEF à partir des volumes de chablis et des données IFN (ancienne méthode, mise à jour). Pour les années intermédiaires, ces valeurs sont estimées en utilisant l'évolution annuelle sur 2001-2006. La tendance de mortalité sur la période 2000-2004 est prolongée sur 2005-2006. Enfin, les branches sont ajoutées grâce aux coefficients de Pignard (1994).].

Les variations de stocks de bois sur pied entre l'ouverture et la fermeture du compte sont mesurées à partir des volumes sur pied et des flux calculés.

Les prélèvements totaux sont estimés en considérant que le volume sur pied de l'année $n$ est égal au volume sur pied de l'année $n-1$, augmenté de la production ligneuse et diminué de la mortalité, des récoltes commercialisée et non commercialisée ${ }^{(2)}$, des pertes d'exploitations et des autres pertes accidentelles (figure 1).

\section{FIGURE 1}

\section{BILAN ANNUEL DES STOCKS ET FLUX DE BOIS SUR PIED}

$$
\begin{aligned}
\text { Volume }^{a}(n) & =\text { Volume }^{\mathrm{a}}(n-1) \\
& + \text { Production ligneuse }^{\mathrm{a}} \\
& - \text { Mortalité a }^{\mathrm{a}} \\
& - \text { Récolte commercialisée } \mathrm{g} \\
& - \text { Récolte non commercialisée (solde) } \\
& - \text { Pertes d'exploitation }{ }^{d} \\
& - \text { Autres pertes accidentelles }{ }^{\mathrm{d}} \text {. }
\end{aligned}
$$

Pour l'évaluation monétaire, Eurostat recommande la méthode du prix moyen de la récolte, qui consiste à appliquer au bois sur pied un prix moyen, sans tenir compte de l'âge des peuplements.

(2) L'estimation de l'autoconsommation issue de ce bilan peut être biaisée, car étant calculée par solde, elle comprend les éventuelles
erreurs d'estimation des autres termes de l'expression. Ce problème sera réglé quand l'lFN pourra publier des estimations de prélèvements. 
Ce prix moyen est estimé par le LEF, en distinguant les essences et en incluant l'autoconsommation de bois. À partir d'une matrice de passage élaborée par Tessier (2000), on transforme les prix ONF, seule source fiable et rigoureuse, bien que partielle, pour les prix du bois sur pied (3), par essence et classe de diamètre en prix sur pied par essence et par catégorie de la récolte. Faute d'indicateurs communs à l'ensemble des acteurs, les prix ONF ont été extrapolés à l'ensemble des bois.

Ces prix de bois sur pied sont alors appliqués aux volumes récoltés commercialisés $m$ et non commercialisés (valorisés au prix du bois de feu). En divisant cette valeur totale de la récolte par le volume correspondant, on obtient le prix moyen utilisé pour valoriser les bois sur pied. Le fait de prendre en compte l'autoconsommation contrebalance le biais lié à l'utilisation des seuls prix ONF.

TABLEAU IV Stocks et flux, physiques et monétaires, des bois sur pied (2000 et 2006) ${ }^{\mathrm{a}, \mathrm{g}, \mathrm{m}, \mathrm{l}}$

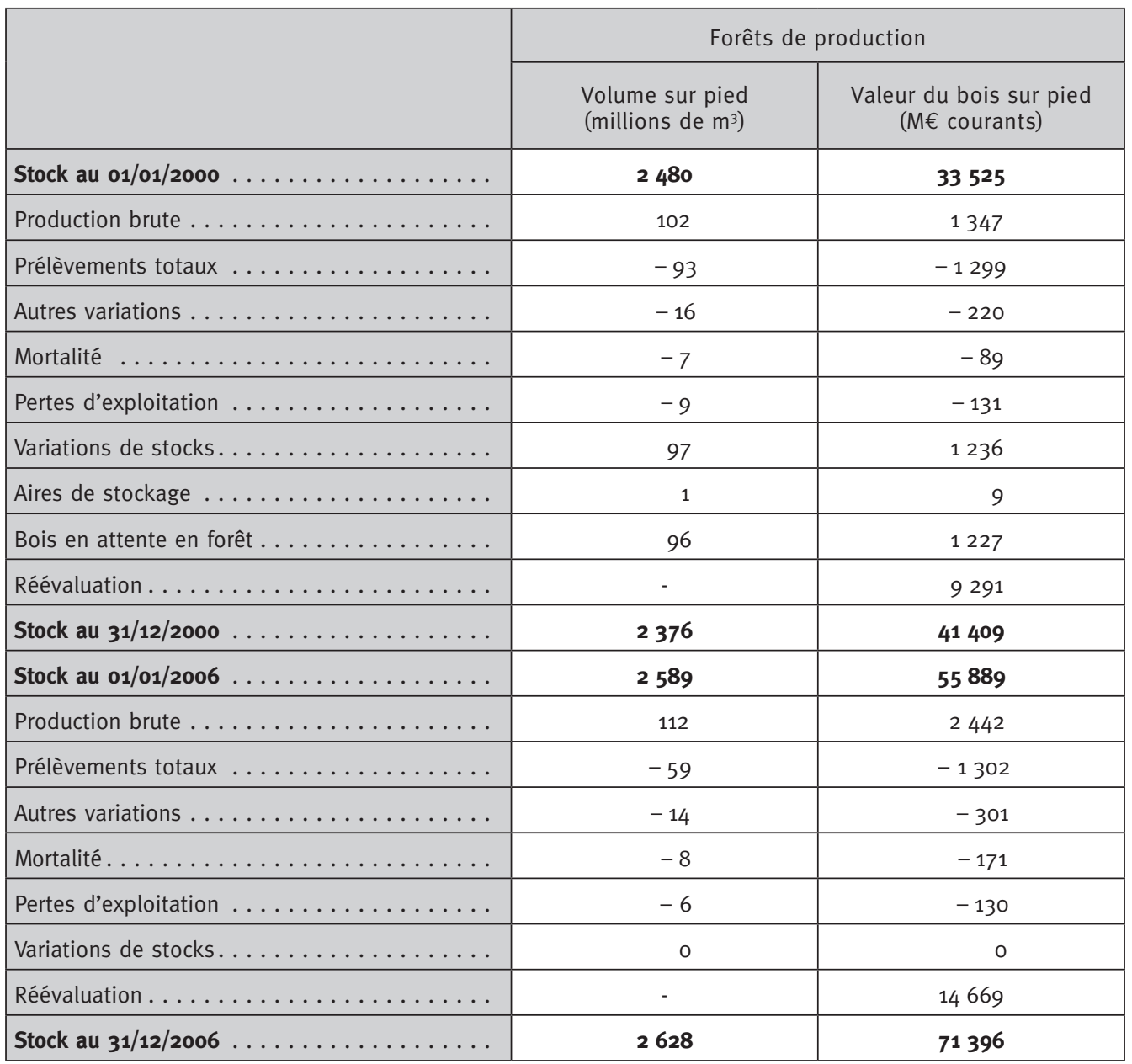

(3) L'INSEE utilisait également les prix ONF dans le Compte économique de la sylviculture (CES) : "Mais c'est la seule source annuelle et régulière disponible, et elle porte sur des bois de qualité relativement constante. De plus, ces prix sont considérés par les professionnels comme une référence pour les ventes privées. " (Rageau, 2002). 
La ligne Réévaluation enregistre les variations de valeur du volume de bois sur pied imputables aux variations de prix entre le début et la fin de période.

Les forêts de production comptent 2628 millions de $\mathrm{m}^{3}$ fin 2006. Si on y ajoute les autres forêts, la France atteint près 2800 millions de $\mathrm{m}^{3}$ de bois sur pied. Ce volume a augmenté de $6 \%$ sur la période considérée. En tenant compte des grosses branches, le volume à l'hectare se monte à $176 \mathrm{~m}^{3} / \mathrm{ha}$ fin 2006, alors qu'il n'était que de $170 \mathrm{~m}^{3} / \mathrm{ha}$ début 2000. Cette tendance peut s'expliquer par plusieurs facteurs :

- les pratiques sylvicoles :

- la conversion du taillis-sous-futaie feuillu en futaie régulière dont le volume à l'hectare est en moyenne $30 \%$ plus élevé (190 contre $148 \mathrm{~m}^{3} / \mathrm{ha}^{\mathrm{e}}$ ) ;

- la plantation d'essences plus productives ;

- la sous-exploitation de la forêt : les bois prélevés ne représentent en moyenne que $63 \%$ de la production biologique nette ;

- le changement climatique : on peut penser que la concentration croissante de $\mathrm{CO}_{2}$ dans l'atmosphère contribue à une augmentation des volumes de bois sur pied.

Les prélèvements totaux et les pertes d'exploitation associées ne représentent en 2006 que $63 \%$ de la production biologique nette de la mortalité. La faible mobilisation des bois fait débat, car si davantage de bois peut théoriquement être récolté, certains obstacles freinent leur mise sur le marché. Si le morcellement de la propriété privée peut trouver des solutions partielles (coopératives, etc.), les coûts qu'impliquerait l'exploitation de certaines zones difficilement accessibles sont des obstacles plus importants.

En 2000, suite aux tempêtes, 176 millions de $\mathrm{m}^{3}$ de bois ont été abattus. Les prélèvements totaux sont constitués à près de $88 \%$ de bois de chablis. Les bois de tempête ont donc été consommés pour partie en 2000 , le reste étant stocké pour être utilisé ensuite. Les stocks de chablis comptabilisés en 2000 comprennent non seulement les bois utilisés en 2001 (prélèvements commercialisés ou non et pertes d'exploitation correspondantes) mais également près de 36 millions de $\mathrm{m}^{3}$ qui n'ont pu être mobilisés, faute de moyens adéquats ou à cause de coûts d'exploitation excessifs, et sont restés en forêt : ces pertes exceptionnelles ont été enregistrées en 2001. Pour ces raisons, la France a connu en 2000 et 2001 des récoltes commercialisées de $30 \%$ et $10 \%$ supérieures environ à celle de 1999. Après un retour à la normale, on peut constater que la récolte commercialisée augmente de $2 \%$ /an depuis 2003. La récolte autoconsommée annuelle est de l'ordre de 22 millions de $\mathrm{m}^{3}$, soit un tiers des prélèvements totaux.

Le prix moyen de la récolte estimé par le LEF appliqué aux volumes sur pied est plus faible que celui utilisé par l'ONF, car il prend en compte l'autoconsommation, considérée comme du bois de feu ne passant pas par les circuits de commercialisation. Ce prix moyen est évalué à 22 euros $/ \mathrm{m}^{3}$ en 2006 : il a donc dépassé le niveau avant tempêtes (20,5 euros $/ \mathrm{m}^{3}$ en 1999 et 12,6 euros $/ \mathrm{m}^{3}$ en $2000^{\mathrm{e}, \mathrm{g}, \mathrm{l}}$ ). Le lien entre l'évolution constatée avec une demande de plus en plus forte, que ce soit pour l'énergie, l'industrie ou la construction, reste encore à établir.

La constante progression du volume sur pied couplée à l'augmentation du prix moyen sur pied des bois engendre un accroissement de la valeur des peuplements.

\section{Les comptes de défoliation}

L'état sanitaire des forêts est appréhendé à partir de la défoliation des forêts (tableau V, p. 550). 


\begin{tabular}{|c|c|c|c|c|}
\hline & \multicolumn{2}{|c|}{ \% Défoliation > $25 \%$} & \multicolumn{2}{|c|}{ Volume sur pied (millions de $\mathrm{m}^{3}$ ) } \\
\hline & 2000 & 2006 & 2000 & 2006 \\
\hline Résineux........... & 12,0 & 23,7 & 103159 & 211569 \\
\hline Feuillus. . . . . . . . & 21,6 & 41,9 & 304970 & 619154 \\
\hline Total. . . . . . . . . . . . & 18,3 & 35,6 & 408129 & 830723 \\
\hline
\end{tabular}

Sont comptabilisés pour les années 2000 et 2006 le pourcentage d'arbres (feuillus et résineux) présentant au moins $25 \%$ de feuilles ou aiguilles perdues, et l'estimation du volume sur pied correspondant.

Le taux de défoliation des arbres a presque doublé sur la période 2000-2006. Les conifères sont moins touchés que les feuillus, mais l'écart se resserre. En 2000, $12 \%$ des conifères étaient défoliés à plus de $25 \%$ contre $22 \%$ des feuillus (soit en moyenne $18 \%$ toutes essences confondues). En 2006, ces taux sont respectivement de $24 \%$ et $42 \%$ (environ $36 \%$ en moyenne). L'aggravation semble s'être ralentie depuis 2005. Ces tendances s'observent dans toute l'Europe et s'expliquent par les tempêtes de 1999 qui ont affaibli une partie des forêts, les rendant plus vulnérables aux parasites et maladies, engendrant une perte de feuilles ou d'aiguilles, et par une année 2003 exceptionnellement chaude et sèche. Les températures anormalement élevées de mars à octobre 2003 et les précipitations rares et peu abondantes ont nettement réduit les apports en eau à la végétation. En réponse à cette sécheresse, les arbres ont perdu leurs feuilles pour mieux résister. À noter que les effets de perturbations climatiques peuvent être plus ou moins grands, selon les essences et le type de sol, et décalés dans le temps. Les polluants atmosphériques, l'acidification des sols et le développement de pathogènes peuvent également jouer dans l'évolution du déficit foliaire.

\section{LES COMPTES EN CARBONE}

\section{Bilan carbone de la biomasse forestière}

Il comptabilise les stocks et flux de carbone dans la biomasse ligneuse (bois, écorce, branches, souches et racines des arbres vivants et morts, arbustes et buissons) d'après les surfaces et volumes renseignés dans les comptes de patrimoine (tableau VI, p. 551).

La variation du stock de carbone provient de deux sources : la variation de la surface boisée et la variation du volume de bois sur pied. Ces deux types de flux sont estimés par application de coefficients issus du projet multidisciplinaire Carbofor (GICC et al., 2004).

En moyenne, sur 2000-2006, le stock de carbone dans le volume sur pied des forêts françaises s'est accru de $4 \% ; 79 \%$ du stock de carbone est contenu dans le bois sur pied et $21 \%$ dans la partie souterraine. Dans les forêts de production, les feuillus représentent $71 \%$ du carbone aérien stocké, grâce à un volume sur pied important et à leurs branches.

\section{Bilan carbone de l'écosystème forestier}

Le tableau VII (p. 551) synthétise les stocks et flux annuels de carbone dans tout l'écosystème forestier (bois sur pied, autre biomasse ligneuse, autre biomasse forestière et sols), calculés en 
TABLEAU VI

Bilan carbone de la biomasse forestière ${ }^{a, b}$

\begin{tabular}{|c|c|c|c|c|c|c|c|c|c|c|c|c|}
\hline \multirow{2}{*}{$\begin{array}{l}\text { MtC } \\
\text { Biomasse forestière } \\
\text { ligneuse }\end{array}$} & \multicolumn{2}{|c|}{$\begin{array}{c}\text { Stock à } \\
\text { l'ouverture }\end{array}$} & \multicolumn{2}{|c|}{$\begin{array}{l}\text { Production } \\
\text { brute }\end{array}$} & \multicolumn{2}{|c|}{$\begin{array}{l}\text { Prélèvements } \\
\text { totaux }\end{array}$} & \multicolumn{2}{|c|}{$\begin{array}{c}\text { Autres } \\
\text { variations }\end{array}$} & \multicolumn{2}{|c|}{$\begin{array}{c}\text { Modification } \\
\text { d'usage / } \\
\text { statut }\end{array}$} & \multicolumn{2}{|c|}{$\begin{array}{l}\text { Stock à la } \\
\text { fermeture }\end{array}$} \\
\hline & 2000 & 2006 & 2000 & 2006 & 2000 & 2006 & 2000 & 2006 & 2000 & 2006 & 2000 & 2006 \\
\hline Bois sur pied. . . . . & 879533 & 915682 & 31073 & 36235 & -31527 & -19071 & -36327 & -4375 & ND & ND & 842752 & 928470 \\
\hline Forêts de production & 825789 & 862225 & 33324 & 36555 & -31527 & -19071 & -36327 & -4375 & ND & ND & 791259 & 875333 \\
\hline Résineux........ & 238767 & 247943 & 11593 & 12582 & -6362 & -7376 & -15267 & -1788 & ND & ND & 228731 & 251361 \\
\hline Feuillus. . . . . . . . & 587023 & 614282 & 21730 & 23973 & -25166 & -11696 & -21059 & -2587 & ND & ND & 562528 & 623972 \\
\hline $\begin{array}{l}\text { Forêts } \\
\text { non productives. . . . }\end{array}$ & 53743 & 53457 & -2250 & -320 & 0 & 0 & 0 & 0 & ND & ND & 51493 & 52761 \\
\hline Autre biomasse bois & 236111 & 246505 & 9573 & 10500 & -8968 & -5497 & 0 & -1262 & -10486 & ND & 226229 & 250246 \\
\hline Total. & 1115644 & 1162187 & 42896 & 47055 & -40495 & -24569 & -36327 & -5637 & -12736 & -1116 & 1068981 & 1178340 \\
\hline
\end{tabular}

ND : Donnée non disponible.

TABLEAU VII

Bilan carbone de l'écosystème forestier ${ }^{a, b, c}$

\begin{tabular}{|c|c|c|c|c|c|c|}
\hline Année & \multicolumn{3}{|c|}{2000} & \multicolumn{3}{|c|}{2006} \\
\hline $\mathrm{MtC}$ & $\begin{array}{c}\text { Stock à } \\
\text { l'ouverture }\end{array}$ & $\begin{array}{l}\text { Variation } \\
\text { de stock }\end{array}$ & $\begin{array}{l}\text { Stock à la } \\
\text { fermeture }\end{array}$ & $\begin{array}{c}\text { Stock à } \\
\text { l'ouverture }\end{array}$ & $\begin{array}{l}\text { Variation } \\
\text { de stock }\end{array}$ & $\begin{array}{l}\text { Stock à la } \\
\text { fermeture }\end{array}$ \\
\hline \multicolumn{7}{|l|}{ Écosystème forestier } \\
\hline Bois sur pied. . . . . . . . . . . . & 879533 & -36781 & 842752 & 915303 & 12791 & 928094 \\
\hline Autre biomasse bois . . . . . . & 236111 & -9881 & 226229 & 246505 & 3741 & 250246 \\
\hline Autre biomasse en forêt . . . . . . & 96934 & 363 & 97297 & 99133 & 371 & 99504 \\
\hline Sols forestiers $\ldots \ldots \ldots \ldots \ldots$ & 1217262 & 4558 & 1221819 & 1244865 & 4661 & 1249526 \\
\hline Total. . . . . . . . . . . . & 2429840 & -41742 & 2388098 & 2505806 & 21564 & 2577370 \\
\hline \multicolumn{7}{|l|}{ Produits bois dans l'économie } \\
\hline Matériaux de construction. .... & 53425 & 268 & 53694 & 55184 & 409 & 55593 \\
\hline Ameublement bois. . . . . . . . & 21128 & 195 & 21323 & 22688 & 390 & 23077 \\
\hline Papier. . . . . . . . . . . . . . & 6126 & 110 & 6236 & 7009 & 221 & 7230 \\
\hline Autres produits bois. . . . . . . & 14017 & 76 & 14093 & 14628 & 153 & 14781 \\
\hline Total. . . . . . . . . . . . . & 94696 & 650 & 95346 & 99509 & 1173 & 100681 \\
\hline
\end{tabular}

Note : Les valeurs étant arrondies, certains totaux sont seulement approchés. 
appliquant les coefficients de Pignard et Dupouey (2002) à la surface des forêts, ainsi que dans les produits bois (matériaux de construction, ameublement, papier et autres produits bois).

Le sol forestier stocke en moyenne 79 tC/ha, la végétation au sol $1 \mathrm{tC} / \mathrm{ha}$. La capacité de stockage par les arbres (parties aérienne et souterraine) varie selon les essences.

Fin 2006, 159 tC/ha sont stockées en moyenne dans les écosystèmes forestiers, soit en tout plus de 2,5 milliards de tonnes de carbone (figure 2, ci-dessous)

FIGURE 2

LE CARBONE STOCKÉ DANS L’ÉCOSYSTÈME FORESTIER a, b

1000 tC

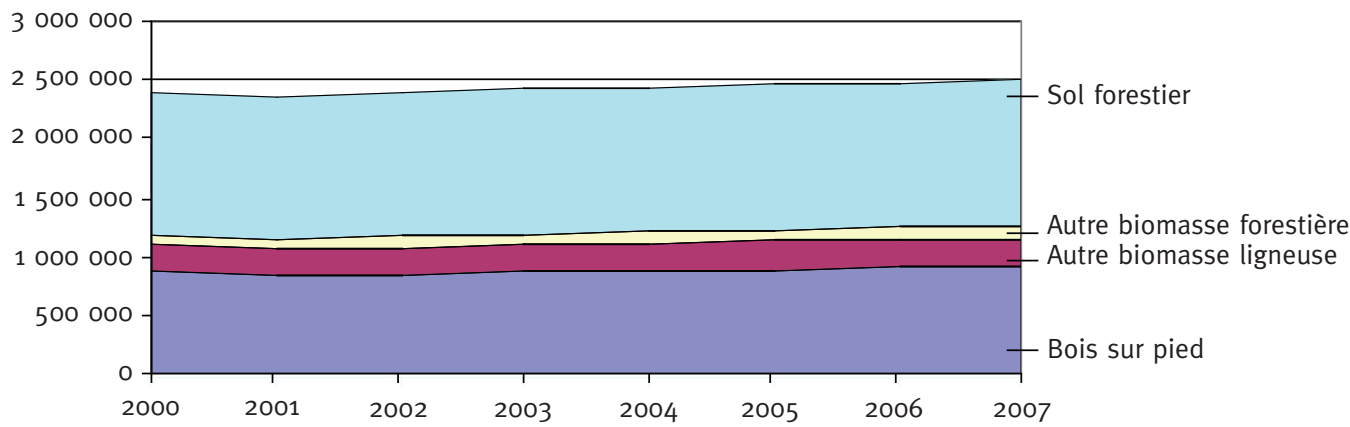

Le stock de carbone a augmenté de $4 \%$ entre 2000 et 2006 . Le stock de carbone dans le bois sur pied et dans la biomasse ligneuse s'est accru de $6 \%$ sur la période, contre seulement $3 \%$ pour le stock de carbone contenu dans les sols et la végétation forestière.

Claire MONTAGNÉ - Alexandra NIEDZWIEDZ

Laboratoire d'économie forestière

UMR 356 INRA AgroParisTech-ENGREF

14 rue Girardet - CS 14216

F-54042 NANCY cedex

(montagne@nancy-engref.inra.fr)

(niedzwiedz@nancy-engref.inra.fr)

NDLR : La deuxième partie de l’article paraîtra dans le numéro 6-2010. 


\section{BIBLIOGRAPHIE}

CMPFE. - Déclaration de Varsovie, $5^{\mathrm{e}}$ conférence ministérielle pour la protection des forêts en Europe, 5-7 novembre 2007. - 2007. Site internet : http://www.foresteurope.org/filestore/foresteurope/Conferences /Varsaw/textes_Varsovie.pdf

EUROPEAN COMMISSION. - The European Framework for Integrated Environmental and Economic Accounting for Forests. IEEAF. Methods and Nomenclatures. Eurostat. Theme 2 : Economy and Finance. - Luxembourg : Office for Official Publications of the European Communities, 2002a. - 106 p.

EUROPEAN COMMISSION. - The European Framework for Integrated Environmental and Economic Accounting for Forests. Results of Pilot Applications. Studies and Research. Eurostat. Theme 2 : Economy and Finance. - Luxembourg : Office for Official Publications of the European Communities, 2002b. - $57 \mathrm{p}$.

GICC, GIP ECOFOR, INRA. - Rapport final du projet Carbofor. Séquestration de Carbone dans les grands écosystèmes forestiers en France. - 2004. - 136 p.

LOCHU (S.). - Évaluation des quantités de carbone stocké. Rapport à la Mission interministérielle de l'effet de serre. - octobre 1998. - $94 \mathrm{p}$.

MONTAGNÉ (C.), NIEDZWIEDZ (A.). - Comptes intégrés économiques et environnementaux de la forêt en France : méthodologie et analyse pour la période 2000-2006. Rapport final SOeS-MEEDDAT. - 2009.

PIGNARD (G.). - Estimation des prélèvements de bois dans la forêt française ; approche forestière de l'autoconsommation. - IFN - ADEME, 1994. - $92 \mathrm{p}$.

PIGNARD (G.), DUPOUEY (J.-L.). - Les flux de carbone dans les forêts françaises et européennes : apport des inventaires forestiers. - Comptes rendus des séances de l'Académie d'Agriculture de France, vol. 88, $\mathrm{n}^{\circ}$ 5, 2002, pp. 7-17.

RAGEAU (F.). - Le Compte économique de la sylviculture et de l'exploitation forestière en base 95. Série

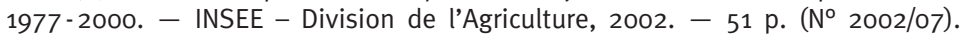

RÉPUBLIQUE FRANÇAISE. - Loi no 2001-602 du 9 juillet 2001 d'orientation sur la forêt. - Journal officiel, $n^{0} 159,11$ juillet 2001, p. 11001 et suivantes.

STIGLITZ (J.E.), SEN (A.), FITOUSSI (J.-P.). - Rapport de la Commission sur la mesure des performances économiques et du progrès social. - septembre 2009. - $324 \mathrm{p}$.

TESSIER (A.). - Comptabilité économique de l'environnement : les comptes de la forêt française. - Études et travaux avec l'IFEN. Document de travail, décembre 2000. - $118 \mathrm{p}$.

UNECE/FAO TBFRA. - Forest Resources of Europe, CIS, North America, Australia, Japan and New Zealand, Main report. - New York and Geneva : UNECE/FAO, 2000. - 445 p.

UNITED NATIONS. - Handbook of National Accounting. Integrated Environmental and Economic Accounting. - New-York : United Nations. - Studies in Methods, Series F, vol. 61, 1993.

UNITED NATIONS. - Revised Handbook for Integrated of National Accounting. Environmental and Economic Accounting. - New York : United Nations. - Studies in Methods, Series F, vol. 61, Rev. 1, 2003.

\section{comptes intéghés Économioues et enuihonnementaux de Lh fohêt en farnce. Première Partie [Résumé]}

L'objet de la comptabilité intégrée économique et environnementale est la prise en compte dans une structure comptable rigoureuse et harmonisée des flux et stocks physiques et monétaires liés à la forêt et au bois. Les comptes de la forêt permettent de cerner la dimension spatiale de la forêt (comptes en surface), de confronter le prélèvement à l'accroissement de la ressource (comptes en volume), d'intégrer les biens et services produits par le secteur forestier (tableaux emplois et ressources) et de mesurer le carbone stocké dans l'écosystème forestier (comptes en carbone). L'ensemble de ces données existe à la fois en termes physiques et monétaires. La rigueur, la périodicité et l'harmonisation de ces tableaux au niveau international contribuent à un meilleur suivi des ressources et des activités. Ils constituent donc un excellent outil pour structurer l'information ainsi que pour l'analyse et l'évaluation des décisions privées ou publiques. La méthodologie d'élaboration des comptes de la forêt selon le format européen préconisé par Eurostat et les principaux résultats pour la période 2000-2006 sont détaillés dans un rapport complet (Niedzwiedz et Montagné, 2009). 


\section{INTEGRATED ECONOMIC AND ENUIRONmENTRL ACCOUNTS FOR FORESTRY IN FRANCE. Part I [Rbstract]}

Integrated economic and environmental accounting allows for a comprehensive description of stocks and flows for the forest and forest-related sector. Forest accounts take into consideration both surface area and standing timber of the forest (forest balance). They integrate goods and services produced by the industries (supply-use tables) and measure the carbon stored by the entire forestry ecosystem (carbon balance). This set of data is available in physical and monetary terms. The harmonization and periodicity of these tables on a European scale contribute to better monitoring of resources and activities. It is an excellent tool for structuring information used in analysing and assessing public and private policies. The methodology for these forest accounts under the European standard (Eurostat) together with the main results for the 20002006 period are detailed in a full report (Niedzwiedz and Montagné, 2009). 